\title{
Influencia de las condiciones de proceso en las características físicas de masas de yuca
}

\author{
Eduardo Rodríguez-Sandoval*, Gérard Cuvelier**, Alejandro Fernández-Quintero*\$ \\ * Escuela de Ingeniería de Alimentos, Universidad del Valle, Cali, Colombia \\ ** UMR SCALE Laboratoire de Biophysique des Matériaux Alimentaires, \\ Ecole Nationale Supérieure des Industries Agricoles et Alimentaires (ENSIA), Massay, France \\ $\S$ e-mail: alfernan@univalle.edu.co
}

(Recibido: Abril 26 de 2006 - Aceptado: Febrero 5 de 2007)

\section{Resumen}

Las propiedades texturales de masas preparadas a partir de harina de yuca precocida fueron estudiadas con el fin de determinar el efecto de las condiciones de proceso en sus características físicas. El módulo de deformabilidad, la dureza, la cohesividad y la adhesividad de masas reconstituidas de harina de yuca se evaluaron utilizando un analizador de textura. Trozos de parénquima de yuca procesados a diferentes condiciones de cocción y almacenados a baja temperatura $\left(-5^{\circ} \mathrm{Cy}-20^{\circ} \mathrm{C}\right)$ por $24 \mathrm{~h}$ se emplearon para elaborar las harinas. Cuando la temperatura en el período de almacenamiento del parénquima cocinado disminuyó de $-5^{\circ} \mathrm{Ca}-20^{\circ} \mathrm{C}$, el módulo de deformabilidad, la dureza y la cohesividad de la masa aumentaron significativamente. Se concluye que la temperatura en el período de reposo del parénquima cocinado es el factor más importante que afecta las propiedades texturales de la masa de yuca.

Palabras clave: Masa de yuca, Harina de yuca, Textura, Módulo de deformabilidad, Almacenamiento en congelación.

\section{Influence of processing conditions on the physical characteristics of cassava doughs}

\begin{abstract}
Textural properties of doughs made from precooked cassava flour were studied to determine the effect of processing conditions on the physical characteristics of cassava doughs. Deformability modulus, hardness, cohesiveness and adhesiveness of reconstituted cassava doughs were evaluated using a texture analyzer. Cassava parenchyma pieces, processed under various cooking conditions and stored at low temperatures $\left(-5^{\circ} \mathrm{C}\right.$ and $\left.-20^{\circ} \mathrm{C}\right)$ for $24 \mathrm{~h}$, were used to make flour. As the resting period temperature of cooked parenchyma decreased from $-5^{\circ} \mathrm{C}$ to $-20^{\circ} \mathrm{C}$, deformability modulus, hardness and cohesiveness of the doughs increased significantly. It is concluded that the temperature during the resting period of cooked cassava parenchyma is the most important factor affecting the textural properties of cassava doughs.
\end{abstract}

Keywords: Cassava dough, Cassava flour, Texture, Deformability modulus, Frozen storing. 


\section{Introducción}

La yuca (manihot esculenta Crantz) es un cultivo alimenticio consumido principalmente en países tropicales (Asaoka et al., 1992). La yuca contribuye al estatus de seguridad alimenticia de poblaciones rurales y es una materia prima para el desarrollo agroindustrial. La industrialización de la yuca sufre múltiples problemas, entre los cuales, se destacan las amplias variaciones de calidad de la materia prima, la falta de tecnología de procesamiento y la fluctuación de precios (Henry \& Gottret, 1998). A medida que la yuca se convierte en un importante cultivo agroindustrial, la logística de suministro de raíces de yuca frescas a las plantas de procesamiento es cada vez más crítica (Thro et al., 1996). Una de las alternativas para superar estos problemas es transformar las raíces frescas en harina de yuca precocida, utilizándola como materia prima para la elaboración de productos con alto valor agregado como croquetas, hojuelas fritas o snacks.

Las masas de materiales ricos en almidón, como cereales, raíces o tubérculos, pueden considerarse como dispersiones de almidón gelatinizado constituidas por gránulos hinchados y fragmentos granulares dispersos en una matriz biopolimérica continua (Eliasson, 1986). El objetivo de cualquier proceso para la obtención de harinas instantáneas es mantener el almidón, que sale del tejido celular, tan bajo como sea posible para evitar una textura pegajosa de los productos reconstituidos con la harina, tales como masas o purés (Lamberti et al., 2004). Investigaciones en el procesamiento de papa para la obtención de hojuelas han demostrado los efectos benéficos de incluir un período de reposo a baja temperatura inmediatamente después de la etapa de calentamiento o precocción del parénquima, resultando un producto más granular y con menor contenido de almidón libre (Lamberti et al., 2004; Ooraikul et al., 1974).

Lamberti et al. (2004) examinaron la transformación del almidón durante el procesamiento de purés preparados por reconstitución de hojuelas de papa y encontraron que el comportamiento reológico del puré de papa dependió de la extensión de la cohesión celular, de la integridad celular y de la cantidad y composición del almidón extracelular en la fase continua. Hopkins \& Gormley (2000) evaluaron el efecto de la congelación de granos de almidón "in situ", es decir dentro del tejido celular, en sus propiedades físicas y mostraron que la congelación del almidón incrementó el grado de retrogradación, la estabilidad de la pasta cocinada y produjo geles con mayor fuerza compresiva aunque con bajos valores de esfuerzo de corte y adhesividad.

En el caso de la yuca se han investigado las propiedades texturales de pastas o purés empleando emulsificantes para evitar una textura pegajosa. Los emulsificantes como monoestearato de glicerilo (GMS) y estearil lactato de sodio (SSL) disminuyen la pegajosidad de purés reconstituidos con harinas de yuca fermentada y con hojuelas de yuca procesadas en un secador de rodillos (Muzanila et al., 2001; Numfor et al., 1998). Adicionalmente, se han llevado a cabo estudios de la conversión del almidón y el tamaño de partícula de la harina de agbelima, una denominación de la masa de yuca fermentada ampliamente consumida en África (Dziedzoave et al., 1999; Sefa-Dedeh, 1989). A pesar de los estudios sobre los efectos de los ingredientes, especialmente los emulsificantes, en las propiedades texturales de masas de yuca, todavía no se ha investigado la influencia de las condiciones de proceso sobre la textura de las masas.

En investigaciones preliminares, se observó la influencia del método y tiempo de cocción, y de las condiciones del periodo de reposo del parénquima precocido sobre las propiedades físicas de masas de yuca (Hernandez, 2005). El objetivo del presente estudio fue determinar el efecto de las condiciones de proceso en las propiedades texturales de masas de yuca elaboradas a partir de harinas de yuca precocidas.

\section{Materiales y métodos}

\subsection{Producción de harinas de yuca}

Raíces de yuca (cultivar HMC-1 cosechadas a 10 meses) provenientes de Jamundí, Valle, Colombia, se utilizaron en los ensayos. Las raíces se procesaron inmediatamente después de 
cosechadas siguiendo el proceso descrito en la Figura 1.

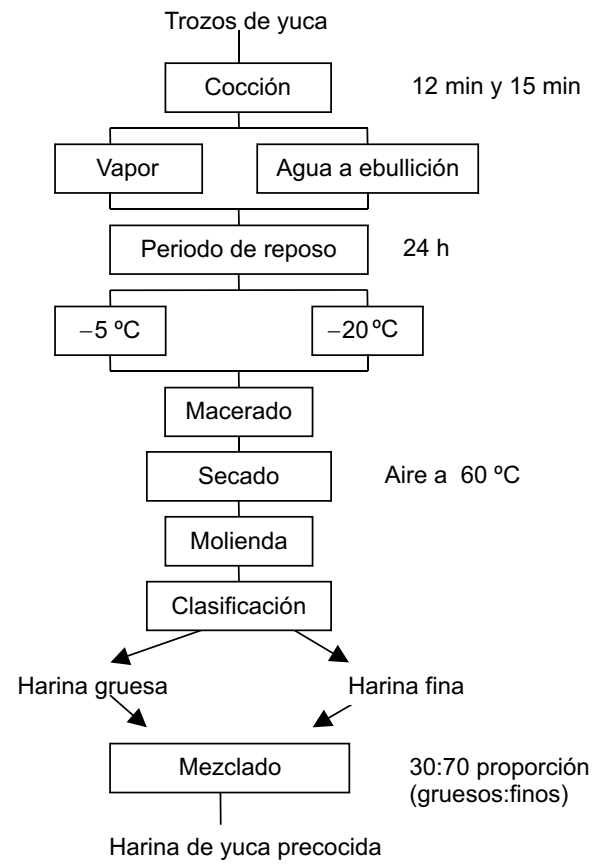

Figura 1. Diagrama de flujo del procesamiento en planta piloto de trozos de parénquima de yuca para la obtención de harina de yuca precocida.

Las raíces de yuca se pelaron manualmente, se cortaron en trozos cilíndricos (altura, $10 \mathrm{~cm}$ ) y se almacenaron en cajas plásticas en una cámara frigorífica (Refrigeración Moderna Ltda., Bogotá, Colombia) a $-20^{\circ} \mathrm{C}$ para su conservación. Muestras de los trozos (3 kg b.h.) se cocinaron utilizando dos métodos de cocción, cocción en vapor en un escaldador (CEV 3/9, Colcocinas, Medellin, Colombia) utilizando bandejas metálicas a una presión de vapor de 15 psi y mediante cocción en agua a ebullición en una marmita (Essen Ltda, Cali, Colombia). Se estudiaron dos tiempos de cocción, 12 min y 15 min. Los trozos cocinados fueron dejados en cámaras frigoríficas $\mathrm{a}-5^{\circ} \mathrm{C}$ y a $-20^{\circ} \mathrm{C}$ por $24 \mathrm{~h}$ y posteriormente macerados en un molino de carnes (P.12N, Mobba, Baladona, Spain). Las muestras maceradas se secaron con aire a $60{ }^{\circ} \mathrm{C}$ en un secador de bandejas hasta un contenido de humedad final de 7-10 \%. El material seco se trituró en un molino de martillos y se clasificó en un tamizador Ro Tap (Model B, W. S. Tyler Inc., Gastonia, N.C., USA) en harina fina $(<300 \mu \mathrm{m}) \mathrm{y}$ harina gruesa $(300 \mathrm{a} 850 \mu \mathrm{m})$. La harina de yuca cocinada se preparó mezclando la harina gruesa y fina en una proporción de 30:70 b. h. (p/p), respectivamente. La proporción de esta mezcla se estableció de acuerdo a estudios realizados en Brasil para la obtención de croquetas de yuca a partir de harina precocida (Berbari, 2001).

\subsection{Preparación de las masas para análisis}

Para formular la rehidratación de la harina en la reconstitución de la masa, el contenido de humedad se determinó secando una muestra de $1 \mathrm{~g}$ en un horno (M. Chopin \& Ciq, Boulogne, France) a $130{ }^{\circ} \mathrm{C}$ por $4 \mathrm{~h}$. El contenido de humedad de la masa de yuca se midió secando una muestra de $2.5 \mathrm{~g}$ en el mismo horno con las condiciones previamente descritas. Se efectuaron determinaciones por duplicado (Fernández, 1996).

Muestras de harina de yuca cocinada (80 g) se rehidrataron a un contenido de agua final de $65 \%$ (b. h.) El agua se agregó a la mezcla de harina y se dejó reposar por $60 \mathrm{~s}$. Luego, se mezclaron en un mezclador (Model 5KPM5, Kitchenaid Inc., St Joseph, Michigan, USA) equipado con un agitador plano durante 60 s para la obtención de la masa.

Las muestras de masa se moldearon en forma cilíndrica colocando $10 \mathrm{~g}$ de masa en un molde de fibra de vidrio cilíndrico ( $35 \mathrm{~mm}$ de diámetro) y aplicando una fuerza de $60 \mathrm{~N}$ durante $120 \mathrm{~s}$ mediante un analizador de textura (TAXT2i, Stable Micro Systems, Surrey, UK) equipado con una celda de carga de $25 \mathrm{~kg}$ y un accesorio cilíndrico. La masa cilíndrica (diámetro $35 \mathrm{~mm}$, altura 8.5-9.5 mm) se retiró cuidadosamente del molde, colocándolo inmediatamente en un contenedor plástico para evitar su deshidratación. Antes de cada ensayo, las masas se dejaron en el contenedor plástico por $20 \mathrm{~min}$ para liberar la energía residual producida durante la preparación de la muestra (Campanella \& Peleg, 1987; Limanond et al., 1999).

\subsection{Método de compresión lubricada}

El ensayo de compresión lubricada se realizó empleando el analizador de textura descrito previamente (TAXT2i, Stable Micro Systems, 
Surrey, UK) equipado con una celda de carga de $25 \mathrm{~kg}$ y un accesorio cilíndrico para compresión (diámetro $100 \mathrm{~mm}$ ). La masa cilíndrica se colocó en una base plana de aluminio y se comprimió 5 $\mathrm{mm}$ de su altura inicial (nivel de deformación $55 \%$ ) con una velocidad de $0.5 \mathrm{~mm} / \mathrm{s}$. La muestra, la base y el accesorio se lubricaron con aceite de parafina para minimizar los efectos friccionales y para asegurar únicamente deformación extensional (Limanond et al., 1999; Osorio et al., 2003). Cada ensayo se realizó por triplicado a temperatura ambiente $\left(24 \pm 1^{\circ} \mathrm{C}\right)$. donde, en la Ec. (1), $\varepsilon_{h}$ es la deformación aplicada, $h_{o}$ es la altura inicial de la muestra $(\mathrm{m})$ y $h(t)$ es la altura de la muestra $(\mathrm{m})$ en cualquier momento de la prueba de compresión.

En la Ec.(2), $F(t)$ es la fuerza perpendicular $(N)$ aplicada al área del material en cualquier momento de la prueba de compresión y $\sigma(t)$ es el esfuerzo aplicado $(\mathrm{Pa})$.

Asumiendo que el material es incompresible, el volumen es constante y así se cumple que:

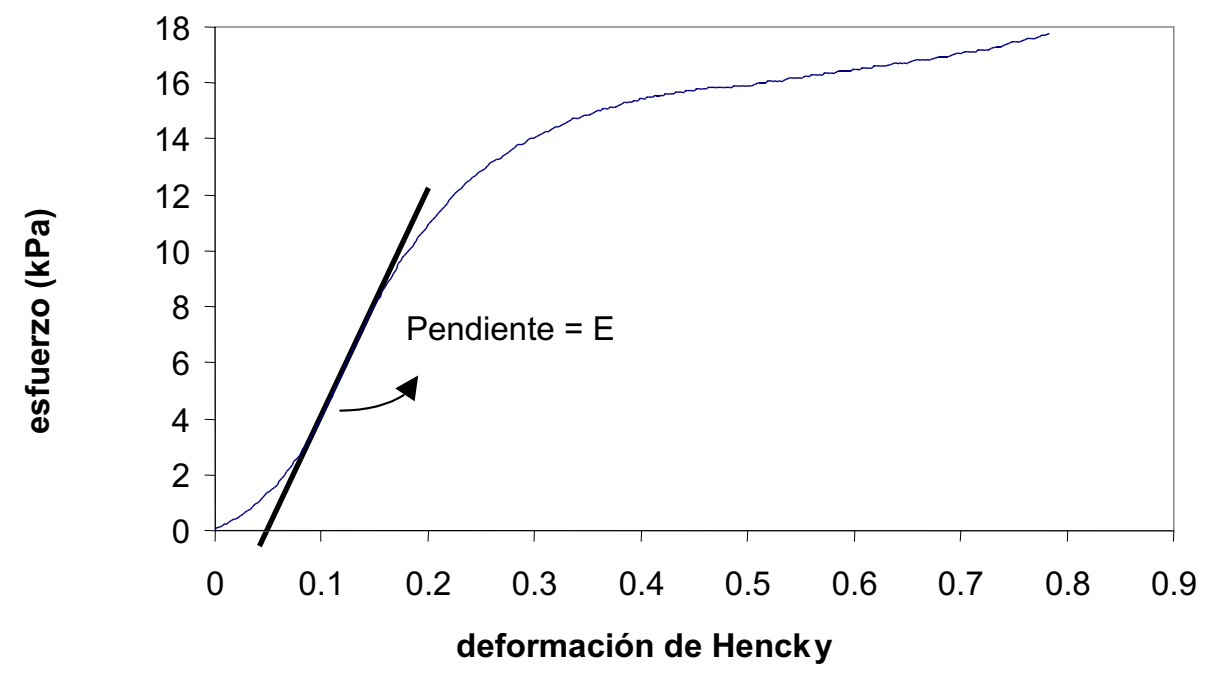

Figura 2. Curva experimental típica de esfuerzo vs. deformación de masa reconstituida de yuca.

Las curvas experimentales de esfuerzo vs. deformación (Figura 2) se generaron a partir de los datos de fuerza-desplazamiento obtenidos en la prueba de compresión, donde la deformación y el esfuerzo se calcularon de acuerdo a la Ecs. (1) y (2), respectivamente:

$$
\begin{gathered}
\varepsilon_{h}(t)=\ln \left|\frac{h_{0}}{h(t)}\right| \\
\sigma(t)=\frac{F(t) h(t)}{A_{0} h_{0}}
\end{gathered}
$$

$$
A(t) h(t)=A_{0} h_{0}
$$

En la Ec.(3), $A_{0}$ es el área inicial de la sección transversal de la muestra $\left(\mathrm{m}^{2}\right)$ y $A(t)$ es el área de la muestra en cualquier momento del ensayo de compresión $\left(\mathrm{m}^{2}\right)$.

El modulo de deformabilidad $(E)$ se obtuvo a partir de la pendiente de la porción lineal de la curva de esfuerzo vs. deformación como se muestra en la Figura 2 (Ak \& Gunasekaran, 1995; Brennan \& Sodah-Ayernor, 1973; Mammarella et al., 2002; Steffe, 1996). Esta curva no fue lineal al comienzo del ensayo probablemente porque la muestra no tuvo una superficie perfectamente lisa; por consiguiente, se presentó un período de transición cuando el accesorio entró en contacto con la superficie de la masa. 


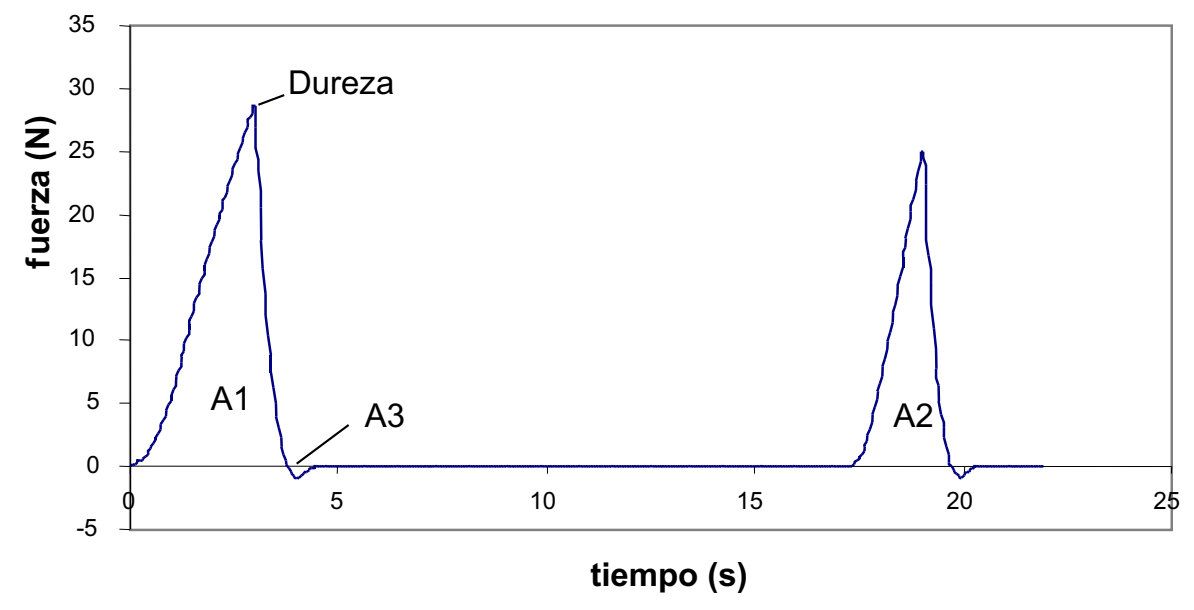

Figura 3. Curva experimental típica del análisis de perfil de textura (TPA) de masa de yuca reconstituida.

\subsection{Análisis de perfil de textura (TPA)}

El análisis de perfil de textura (TPA) se efectuó utilizando el analizador de textura equipado con la misma celda de carga y el mismo accesorio descrito en el ensayo anterior. Un papel de superficie rugosa (papel de lija) se adhirió al accesorio del equipo.

La masa cilíndrica se colocó en la base plana de aluminio y se comprimió $3 \mathrm{~mm}$ de su altura inicial en el primer ciclo de compresión, se sostuvo por $10 \mathrm{~s}$ y se comprimió $1 \mathrm{~mm}$ en el segundo ciclo de compresión. La velocidad de la celda fue de $1 \mathrm{~mm} / \mathrm{s}$.
La muestra, el accesorio y la base no se lubricaron. En cada curva de TPA (Figura 3) se determinaron tres parámetros texturales: la dureza, que es la fuerza máxima durante el primer ciclo de compresión (Bourne, 2002; Jankowski, 1992), la cohesividad, que es una función directa del trabajo requerido para sobrepasar la estructura interna de un producto y que se obtiene por la relación entre el área positiva durante el segundo ciclo de compresión $\left(\mathrm{A}_{2}\right)$ y el área positiva del primer ciclo de compresión $\left(\mathrm{A}_{1}\right)$ (Bourne, 1978; Jankowski, 1992; Schweingruber et al., 1979) y la adhesividad, que es el área negativa del primer ciclo de compresión $\left(\mathrm{A}_{3}\right)$ (Bourne, 2002).

Tabla 1. Factores aplicados en el diseño experimental.

\begin{tabular}{lccccc}
\hline Factor & Unidades & \multicolumn{2}{l}{ Valores reales } & \multicolumn{2}{l}{ Niveles codificados } \\
\hline Tipo de cocción & & agua & vapor & -1 & 1 \\
\hline Tiempo de cocción & $\min$ & 12 & 15 & -1 & 1 \\
\hline Temperatura de reposo & ${ }^{\circ} \mathrm{C}$ & -20 & -5 & -1 & 1 \\
\hline
\end{tabular}

Tabla 2. Coeficientes de la correlación lineal múltiple obtenida para predecir el módulo de deformabilidad, la dureza y la cohesividad de masas reconstituidas de yuca en los tratamientos con agua y vapor.

\begin{tabular}{|c|c|c|c|c|c|c|c|c|c|}
\hline \multirow[b]{2}{*}{ Coeficiente } & \multicolumn{3}{|c|}{$\begin{array}{l}\text { Módulo de deformabilidad } \\
(\mathrm{kPa})\end{array}$} & \multicolumn{2}{|c|}{$\begin{array}{c}\text { Dureza } \\
(\mathrm{N})\end{array}$} & \multicolumn{4}{|c|}{ Cohesividad } \\
\hline & agua & vapor & & agua & vapor & $* *$ & agua & vapor & \\
\hline$a_{0}$ & 128.85 & 126.07 & $* * *$ & 26.75 & 22.13 & $* *$ & 0.04 & 0.03 & $*$ \\
\hline$a_{1}$ & \multicolumn{2}{|c|}{-5.39} & $* * *$ & \multicolumn{2}{|c|}{-0.33} & & \multicolumn{2}{|c|}{0.02} & $*$ \\
\hline$a_{2}$ & \multicolumn{2}{|c|}{-1.05} & $* * *$ & \multicolumn{2}{|c|}{-0.53} & $* * *$ & \multicolumn{2}{|c|}{0.0069} & $* *$ \\
\hline
\end{tabular}

$*$ significante al 5\% $\quad{ }^{* *}$ significante al $1 \% \quad * *$ significante al $0.1 \%$ 
Ingeniería y Competitividad, Volumen 9, No. 1, p. 7 - 17 (2007)

Tabla 3. Módulos de deformabilidad ( $\mathrm{kPa}$ ) de las masas de yuca reconstituidas

\begin{tabular}{cccc}
\hline & Condiciones & & $\begin{array}{c}\text { Módulo de } \\
\text { deformabilidad } \\
(\mathrm{kPa})\end{array}$ \\
$\begin{array}{c}\text { Método de } \\
\text { cocción }\end{array}$ & Tiempo de & Temperatura de \\
& cocción $(\mathrm{min})$ & reposo $\left({ }^{\circ} \mathrm{C}\right)$ & $69.4 \pm 1.2$ \\
Agua & 12 & -5 & $85.1 \pm 0.8$ \\
& \multirow{2}{*}{15} & -20 & $53.2 \pm 1.8$ \\
& \multirow{2}{*}{12} & -5 & $68.9 \pm 0.7$ \\
Vapor & \multirow{2}{*}{15} & -20 & $66.6 \pm 1.7$ \\
& & -20 & $82.3 \pm 2.2$ \\
& & -5 & $50.5 \pm 1.1$ \\
\end{tabular}

*Medida de al menos tres réplicas \pm desviación normal

Tabla 4. Durezas (N) de las masas de yuca reconstituidas

\begin{tabular}{|c|c|c|c|}
\hline \multicolumn{3}{|c|}{ Condiciones } & \multirow{2}{*}{$\begin{array}{c}\text { Dureza* } \\
(\mathrm{N})\end{array}$} \\
\hline $\begin{array}{l}\text { Método de } \\
\text { cocción }\end{array}$ & $\begin{array}{c}\text { Tiempo de } \\
\text { cocción (min) }\end{array}$ & $\begin{array}{c}\text { Temperatura de } \\
\text { reposo }\left({ }^{\circ} \mathrm{C}\right)\end{array}$ & \\
\hline & 12 & $\begin{array}{r}-5 \\
-20\end{array}$ & $\begin{array}{l}25.5 \pm 0.9 \\
33.5 \pm 1.8\end{array}$ \\
\hline Agua & 15 & $\begin{array}{r}-5 \\
-20\end{array}$ & $\begin{array}{l}24.5 \pm 1.5 \\
32.5 \pm 2.1\end{array}$ \\
\hline Vapor & 15 & $\begin{array}{r}-5 \\
-20 \\
-5 \\
-20\end{array}$ & $\begin{array}{l}20.8 \pm 1.7 \\
28.8 \pm 2.3 \\
19.9 \pm 0.8 \\
27.9 \pm 1.9\end{array}$ \\
\hline
\end{tabular}

*Medida de al menos tres réplicas \pm desviación normal

Tabla 5. Cohesividades de las masas de yuca reconstituidas

\begin{tabular}{cccc}
\hline & \multicolumn{2}{c}{ Condiciones } & Cohesividad* \\
\hline $\begin{array}{c}\text { Método de } \\
\text { cocción }\end{array}$ & Tiempo de & Temperatura de & \\
& cocción (min) & reposo $\left({ }^{\circ} \mathrm{C}\right)$ & \\
\multirow{2}{*}{ Agua } & \multirow{2}{*}{12} & -5 & $0.33 \pm 0.5$ \\
& \multirow{2}{*}{15} & -20 & $0.43 \pm 0.3$ \\
& \multirow{2}{*}{12} & -5 & $0.39 \pm 0.7$ \\
Vapor & \multirow{2}{*}{15} & -20 & $0.5 \pm 0.1$ \\
& & -5 & $0.31 \pm 0.6$ \\
& & -20 & $0.42 \pm 0.3$ \\
& & -20 & $0.38 \pm 0.8$ \\
\end{tabular}

*Medida de al menos tres réplicas \pm desviación normal 


\subsection{Análisis estadístico}

En este estudio, el análisis estadístico se desarrolló en el programa Design Expert 6.0 (Stat-Ease Inc., Minneapolis, USA). Se utilizó un diseño factorial fraccionado $2^{\mathrm{k}}$ de tres factores (tipo de cocción, tiempo de cocción y temperatura de reposo) como se muestra en la Tabla 1. Para cada variable dependiente $Y$ (módulo de deformabilidad, dureza y cohesividad) se obtuvo una correlación lineal múltiple $Y=a_{0}+a_{1} X_{1}+a_{2} X_{2}$, donde $X_{1}$ es el tiempo de cocción y $X_{2}$ es la temperatura de reposo. Los valores de los coeficientes de la correlación y sus niveles de significancia se muestran en la Tabla 2.

El análisis de varianza (ANOVA) se utilizó para determinar las diferencias entre tratamientos a un nivel de significancia del 5\% (Montgomery, 1991).

\section{Resultados}

\subsection{Módulo de deformabilidad}

El módulo de deformabilidad $(E)$ de la masa de yuca reconstituida se presenta en la Tabla 3. El valor de $E$ se afectó significativamente $(\mathrm{P}<0.01)$ por el tiempo de cocción y la temperatura a la cual se almacenó el parénquima cocinado por $24 \mathrm{~h}$ (ver Tabla 2). Estos resultados podrían indicar que la harina procesada a partir de parénquima de yuca cocinado y almacenado a $-20^{\circ} \mathrm{C}$ produce una masa con mayor rigidez que la masa preparada con harina obtenida de parénquima de yuca cocinado y reposado a $-5{ }^{\circ} \mathrm{C}$. Para cada temperatura en el periodo de reposo, los valores de $E$ para masas reconstituidas con harina preparada de parénquima cocinado por $12 \mathrm{~min}$ fueron mayores que los reportados para masas preparadas con harina elaborada con un tratamiento de cocción del parénquima por $15 \mathrm{~min}$. Sin embargo, el módulo para masas preparadas con harina cuyo parénquima se cocinó por 12 min y se dejo a $-5^{\circ} \mathrm{C}$ fue similar al de las masas elaboradas con harina de parénquima cocinado por 15 min y almacenado a $-20{ }^{\circ} \mathrm{C}$. El método de cocción no afectó significativamente el módulo de deformabilidad de la masa como se muestra en la Tabla 2.

\subsection{Análisis de perfil de textura}

La dureza y la cohesividad se muestran en las Tablas 4 y 5, respectivamente. Los coeficientes de variación de los resultados de adhesividad fueron mayores al $30 \%$, por lo cual, este parámetro textural no fue tomado en cuenta en este estudio, mientras que los otros parámetros tuvieron coeficientes de variación en el rango de $1.7 \%$ a $14 \%$.

El tiempo de cocción $(\mathrm{P}<0.5)$ y el periodo de reposo a baja temperatura $(\mathrm{P}<0.01)$ afectaron significativamente la cohesividad de la masa de yuca reconstituida (ver Tabla 2). Las masas elaboradas con harina de parénquima cocinado por 12 min y almacenado a $-5^{\circ} \mathrm{C}$ fueron menos cohesivas que las preparadas con harina de parénquima cocinado por 15 min y almacenado a $-20{ }^{\circ} \mathrm{C}$. El método de cocción no afectó significativamente la cohesividad de la masa. En el presente trabajo, la cohesividad de la masa estuvo en el rango de 0.3 a 0.5 . Estos resultados fueron similares a los reportados por Schweingruber et al. (1979) para masas de papa reconstituidas, utilizando una máquina universal de ensayos Instron, los cuales estuvieron en un rango de 0.36 a 0.44. Numfor et al. (1998) encontraron valores de cohesividad de 0.68 a 0.79 para pastas preparadas con harinas nativas y fermentadas de yuca.

\section{Discusión}

La masa de yuca reconstituida podría considerarse como una dispersión compuesta de gránulos hinchados, fragmentos granulares de almidón y componentes menores como fibra, proteína y minerales dispersos en una matriz biopolimérica continua. Las características de esta matriz, la cual consiste principalmente de almidón libre, probablemente son determinantes en las propiedades texturales de la masa de yuca reconstituida. La masa de papa reconstituida con hojuelas precocidas secas se compone principalmente de células enteras y fragmentadas embebidas en una fase de almidón extracelular (Lamberti et al., 2004). 
De acuerdo con Szymonska et al. (2000) y Redmond et al. (2002), la estructura del gránulo de almidón gelatinizado podría sufrir daño durante la congelación debido a la presión ejercida sobre los gránulos por la formación de la matriz de hielo. Este proceso podría liberar mayor cantidad de almidón libre, formando una matriz más elástica en la fase continua. El grado de modificación o la posible destrucción de los gránulos congelados dependen del contenido de agua en la muestra, el tamaño del gránulo y la temperatura aplicada (Hopkins \& Gormley, 2000; Szymonska et al., 2000). Las observaciones anteriores pueden explicar por qué las muestras de masa elaboradas con harina preparada de parénquima de yuca cocinado y almacenado a $-20^{\circ} \mathrm{C}$ por $24 \mathrm{~h}$, tuvieron mayor rigidez que las elaboradas con harina obtenida de parénquima cocinado y reposado a $-5{ }^{\circ} \mathrm{C}$. Por otro lado, el parénquima de yuca cocinado en agua a ebullición presentó un incremento en peso de $4 \%$ debido a la absorción de agua, mientras que el peso del parénquima cocinado en vapor fue casi constante. Esta diferencia podría tomarse en cuenta para explicar los resultados de la dureza de la masa de acuerdo al método de cocción.

La dureza y el módulo de deformabilidad son parámetros que cuantifican la rigidez de un material (Mammarella et al., 2002; Peleg, 1987). Por consiguiente, los resultados de estos parámetros indicaron que la disminución de la temperatura en el período de reposo del parénquima de yuca cocinado de $-5^{\circ} \mathrm{C}$ a $-20^{\circ} \mathrm{C}$ incrementó la firmeza del material. Este comportamiento se debe probablemente al aumento de la fracción de volumen del almidón libre, el cual desarrolla una red elástica en la fase continua dentro del sistema. Jankowski (1992) reportó que el incremento en la consistencia y la dureza de papa cocinada se aceleraron a menores temperaturas de acondicionamiento, lo cual fue una característica del proceso de retrogradación del almidón. La retrogradación se emplea para describir los cambios que ocurren durante el enfriamiento o almacenamiento del almidón gelatinizado (Fredriksson et al., 1998; Karim et al., 2000). En el proceso de retrogradación de los geles de almidón, el desarrollo de la cristalinidad en corto tiempo se atribuye a la cristalización de la fracción de amilosa y los cambios a largo plazo se deben a la fracción de amilopectina (Miles et al., 1985). De acuerdo con estos últimos autores, los cambios en las propiedades mecánicas de los geles de almidón se relacionan con la formación de la red cristalina después de la asociación de la amilosa. Por lo tanto, sería posible interpretar el comportamiento de la masa de yuca reconstituida en términos de retrogradación, la cual se podría reflejar en el incremento de la rigidez de los gránulos de almidón y la matriz biopolimérica por la agregación de amilosa a baja temperatura. Sin embargo, esta hipótesis se debe comprobar realizando un seguimiento a la retrogradación del almidón en el parénquima precocido y reposado a baja temperatura como se describe en este estudio.

El aumento en el módulo de deformabilidad de la masa obtenida con un tratamiento de cocción por 12 min podría atribuirse al contenido y tamaño granular (Hadziyev \& Steele, 1979; Okechukwu \& Rao, 1996; Rolee \& Le Meste, 1997). Si la masa de yuca reconstituida se considera como un material compuesto por gránulos de almidón que actúan como partículas de relleno en una matriz de polisacáridos, las masas con un tratamiento de cocción por 12 min tendrían mayor cantidad de gránulos de almidón intactos que retienen su forma redondeada con poco o ningún hinchamiento comparado con las masas que tuvieron un tratamiento de cocción por $15 \mathrm{~min}$ (Eliasson, 1986). Estos gránulos de almidón intactos son elementos de relleno que tienen interacciones intergranulares, tales como entrecruzamientos entre las moléculas superficiales adyacentes a los gránulos, las cuales podrían producir una mayor rigidez en el material (Evans \& Haisman, 1979).

Durante el calentamiento de una suspensión amilácea se absorbe agua y se libera material del gránulo de almidón al mismo tiempo. En suspensiones de almidón gelatinizado, la amilosa se encuentra fuera del gránulo formando una red alrededor de este (Eliasson \& Gudmundsson, 1996). Al incrementar el tiempo de cocción del parénquima de yuca de $12 \mathrm{~min}$ a $15 \mathrm{~min}$ y al disminuir la temperatura en el periodo de reposo de $-5^{\circ} \mathrm{Ca}-20^{\circ} \mathrm{C}$ se genera una masa de yuca con alta cohesividad, debido al aumento en el contenido de amilosa en la fase continua y a la fuerte interacción entre los gránulos de almidón y la matriz continua. 


\section{Conclusión}

Los resultados de este estudio mostraron que la temperatura en el período de reposo del parénquima de yuca cocinado fue el principal factor que afectó la textura de la masa de yuca reconstituida. Al disminuir la temperatura en el periodo de reposo de $-5^{\circ} \mathrm{Ca}-20^{\circ} \mathrm{C}$ se incrementó el valor del módulo de deformabilidad $(E)$, la dureza y la cohesividad de la masa. Al incrementar el tiempo de cocción del parénquima de yuca de 12 min a 15 min se disminuyó el valor de $E$ y se incrementó la cohesividad de la masa.

La integridad y el tamaño granular, el contenido de amilosa en la fase continua y la interacción entre los gránulos de almidón y la matriz pueden causar este comportamiento. El método de cocción sólo tuvo influencia en la dureza de la masa de yuca. La masa con un tratamiento de cocción en agua a ebullición presentó mayor firmeza que la masa con un tratamiento de cocción en vapor.

La investigación de la transformación del almidón presente en la raíz de yuca durante el procesamiento planteado en este estudio es necesaria para comprender con mayor claridad el efecto de las condiciones de proceso en la textura de la masa de yuca reconstituida.

\section{Agradecimientos}

E. Rodriguez-Sandoval agradece la beca de investigación otorgada por COLCIENCIAS y la Embajada de Francia en Colombia, Programa Bomplan. También se reconoce la colaboración de CLAYUCA por el apoyo logístico y el suministro de las raíces de yuca para el estudio.

\section{Referencias bibliográficas}

Ak, M.M., \& Gunasekaran, S. (1995). Evaluating rheological properties of mozzarella cheese by the squeezing flow method. Journal of Texture Studies 26,(6), 95-712.
Asaoka, M., Blanshard, J.M.V., \& Rickard, J.E. (1992). Effects of cultivar and growth season on the gelatinisation properties of cassava ( $m a n i h o t$ esculenta) $\mathrm{starch}$. Journal of the Science of Food and Agriculture 59 (1), 53-58.

Berbari, S.A.G. (2001). Desenvolvimento de tecnología para obtenção de produto formatado e congelado de mandioca (manihot esculenta Crantz). Tesis de doutorado, Faculdade de Engenharia de Alimentos Tecnología de Alimentos, Universidade Estadual de Campinas, UNICAMP, Brasil.

Bourne, M. (1978). Texture profile analysis. Food Technology 32 (7), 62-66.

Bourne, M. (2002). Food texture and viscosity: concept and measurement. Second Edition, Academic Press, San Diego, California.

Brennan, J.G., \& Sodah-Ayernor, G. (1973). A study of the kinetics of retrogradation in a starchbased dough made from dehydrated yam (Dioscorea rotundata L. Poir). Starch/Stärke 25 (8), 276-280.

Campanella, O.H., \& Peleg, M. (1987). Squeezing flow viscosimetry of peanut butter. Journal of Food Science 52 (1), 180-184.

Dziedzoave, N.T., Ellis, W.O., Oldham, J.H., \& Osei-Yaw, A. (1999). Subjective and objective assessment of 'agbelima' (cassava dough) quality. Food Control 10(2), 63-67.

Eliasson, A.C. (1986). Viscoelastic behaviour during the gelatinization of starch I. Comparison of wheat, maize, potato and waxy-barley starches. Journal of Texture Studies 17 (3), 253-265.

Eliasson, A.C., \& Gudmundsson, M. (1996). Starch: physicochemical and functional aspects. In: A.C. Eliasson (editor), Carbohydrates in food. Marcel Dekker Inc., New York, (Chapter 10). 
Evans, I.D., \&. Haisman, D.R. (1979). Rheology of gelatinised starch suspensions. Journal of Texture Studies 10 (4), 347-370.

Fernández, A. (1996). Effects of processing procedures and cultivar on the properties of cassava flour and starch. Doctoral Thesis, Department of Applied Biochemistry and Food Science (ABFS). University of Nottingham, Nottingham, United Kingdom.

Fredriksson, H., Silverio, J., Andersson, R., Eliasson, A.C., \& Åman, P. (1998). The influence of amylose and amylopectin characteristics on gelatinization and retrogradation properties of different starches. Carbohydrate Polymers 35 (3/4), 119-134.

Hadziyev, D., \& Steele, L. (1979). Dehydrated mashed potatoes: chemical and biochemical aspects. Advances in Food Research 25, 55-136.

Henry, G. \&, Gottret, V. (1998). Client-led agroindustrial action development: The case of cassava starch in Cauca Valley, Colombia. In Proceedings of the AFSR\&E Symposium, Pretoria, South Africa.

http://www.ciat.cgiar.org/agroempresas/pdf/clien tled-agroindustrial_valley_cauca.pdf

Hernández, J. (2005). Estudio comparativo de elaboración de croquetas de yuca (manihot esculenta Crantz) mediante el método convencional industrial y un método que incluye la producción de una harina precocida. Trabajo de grado en ingeniería agroindustrial, Universidad Nacional de Colombia, Palmira, Valle, Colombia.

Hopkings, S., \& Gormley, R. (2000). Rheological properties of pastes and gels made from starch separated from different potato cultivars. LWTFood Science and Technology 33 (5), 388-396.

Jankowski, T. (1992). Influence of starch retrogradation on the texture of cooked potato tuber. International Journal of Food Science and Technology 27 (6), 637-642.

Karim, A.A., Norziah, M.H., \& Seow, C.C. (2000). Methods for the study of starch retrogradation. Food Chemistry 71 (1), 9-36.
Lamberti, M., Geiselmann, A., Conde-Petit, B., \& Escher, F. (2004). Starch transformation and structure development in production and reconstitution of potato flakes. LWT-Food Science and Technology 37 (4), 417-427.

Limanond, B., Castell-Perez, M.E., \& Moreira, R.G. (1999). Effect of time and storage conditions on the rheological properties of masa for corn tortillas. LWT-Food Science and Technology 32 (6), 344-348.

Mammarella, E.J., de Piante Vicín, D.A., \& Rubiolo, A.C. (2002). Evaluation of stress-strain for characterization of the rheological behavior of alginate and carrageenan gels. Brazilian Journal of Chemical Engineering 19 (4), 403-409.

Miles, M.J., Morris, V.J., Orford, P.D., \& Ring, S.G. (1985). The roles of amylose and amylopectin in gelation and retrogradation of starch. Carbohydrate Research 135 (2), 271-281.

Montgomery, D. (1991). Diseño y análisis de experimentos. Grupo Editorial Iberoamericano, México.

Muzanila, Y.C., Brennan, J.G., King, R.D., \& MacDougall, D.B. (2001). Texture of reconstituted cassava flakes. Tropical Science 41 (2), 55-59.

Numfor, F.A., Walter, W.M., \& Schwartz, S.J. (1998). Emulsifiers affect the texture of pastes made from fermented and non-fermented cassava flours. International Journal of Food Science and Technology 33 (5), 455-460.

Okechukwu, P.E., \& Rao, M.A. (1996). Kinetics of cowpea starch gelatinization based on granule swelling. Starch/Stärke 48, 43-47.

Ooraikul, B., Packer, G.J.K., \& Hadziyev, D. (1974). Starch and pectic substances as affected by a freeze-thaw potato granule process. Journal of Food Science 39 (2), 358-364.

Osorio, F., Gahona, E., \& Alvarez, F. (2003). Water absorption effects on biaxial extensional viscosity of wheat flour dough. Journal of Texture Studies 34 (2), 147-157. 
Peleg, M. (1987). The basics of solid foods rheology. In: H.R. Moskowitz (editor), Food texture: instrumental and sensory measurement. Marcel Dekker Inc., New York, p. 3-33.

Redmond, G.A., Butler, F., \& Gormley, T.R. (2002). The effect of freezing conditions on the quality of freeze-chilled reconstituted mashed potato. LWT-Food Science and Technology 35 (2), 201-204.

Rolee, A., \& Le Meste, M. (1997). Thermomechanical behavior of concentrated starch-water preparations. Cereal Chemistry 74 (5), 581-588.

Schweingruber, P., Escher, F., \& Solms, J. (1979). Instrumental measurement of texture of instant mashed potato. In: P. Sherman (editor), Food texture and rheology. Academic Press Inc., London, p. 201-22.

Sefa-Dedeh, S. (1989). Effects of particle size on some physicochemical characteristics of 'agbelima' (cassava dough) and corn dough. Tropical Science 29, 21-32.

Steffe, J.F. (1996). Rheological methods in food process engineering. Second Edition. Freeman Press, East Lansing, Michigan, USA.

http://www.egr.msu.edu/ steffe/freebook/ STEFFE.pdf

Szymonska, J., Krok, F., \& Tomasik, P. (2000). Deep-freezing of potato starch. International Journal of Biological Macromolecules 27 (4), 307-314.

Thro, A.M., Roca, W.M., \& Henry, G. (1996). The cassava biotechnology network and biotechnologies for improving the processing of quality of cassava. In: D. Dufour, G.M. O'Brien, and R. Best (editors), Cassava flour and starch: progress in research and development. CIAT, Cali, (Chapter 3). 